\title{
Do Women Experience the Same Benefits as Men from Implantable Cardioverter Defibrillators?
}

\author{
Douglas S. Lee, for the Ontario ICD Database Investigators
}

\section{The Issue}

Implantable cardioverter defibrillators (ICDs) are used for primary prevention in those who are at risk of sudden cardiac death (SCD) and for secondary prevention in those who have been resuscitated from a cardiac arrest. Several randomized controlled trials have found that treatment with an ICD reduced mortality, and the device has been assumed to be equally effective in women and men (Antiarrhythmics versus Implantable Defibrillators Investigators 1997; Bardy et al. 2005; Moss et al. 2002). Indeed, clinical guidelines do not consider the sex of the patient in their recommendations for ICD implantation. Although ICDs are now routinely implanted in patients regardless of their sex, recent studies have questioned whether there are differences in ICD use and outcomes between women and men.

The weight of evidence suggests that women are less likely than men to undergo ICD implantation. Indeed, landmark randomized controlled trials of ICDs enrolled approximately 75\% men (Bardy et al. 2005; Moss et al. 2002). Data from Ontario indicate that compared with men, far fewer women with myocardial infarction or heart failure have had the device implanted for primary prevention, and this difference has persisted over time (MacFadden et al. 2009). Women are also far less likely to undergo ICD implantation for secondary prevention after cardiac arrest, although the differences in device utilization for post-cardiac arrest indications have been attenuated over time (MacFadden et al. 2009). Although few studies have examined the reasons for differences in ICD use between the sexes, there are several potential explanations, including lower rates of referral of women, reduced likelihood of accepting women for the procedure and women's refusal to undergo defibrillator implantation.

Outcomes for women who undergo ICD implantation have not been fully evaluated. Data from the US National Cardiovascular Disease Registry (NCDR) ICD Registry suggest that women have a higher risk of in-hospital complications than men (Peterson et al. 2009). However, ICD complications are more likely to occur after discharge from hospital, and these events were not explored. In a study of defibrillator-related early complications from the Ontario ICD Database, compli- cation rates were found to be higher than previously anticipated (Lee et al. 2010). Rates of major, minor and any complications at 45 days were $4.1 \%, 5.1 \%$ and $7.4 \%$, respectively. The majority of these events occurred after discharge from the index ICD-implantation admission, as evidenced by the $0.8 \%$ in-hospital complication rate. Importantly, early implant-related

\section{Women were $\mathbf{7 8} \%$ more likely than men to experience a major early complication.}

complications of a surgical nature were associated with a 24-fold increase in mortality in the next six months. Complications due to worsening of the underlying cardiac disease substrate occurring within the first 45 days of ICD implantation conferred a 12 -fold increase in risk of death at six months.

A number of studies have suggested that the risk of SCD is reduced in women. Early epidemiological data from the population-based Framingham Heart Study indicated that women have a lower intrinsic risk of SCD than men (Cupples et al. 1992; Kannel et al. 1998). A small single-centre study of ICD recipients composed of only 59 women and 340 men found that women experienced fewer ventricular tachyarrhythmias; however, the study included a small number of such events and a limited ability to adjust for other clinical factors (Lampert et al. 2004). Other studies comparing SCD risk in women versus men found sex differences in the degree of left ventricular dysfunction, types of cardiovascular disease and arrhythmia inducibility (Albert et al. 1996; Chugh et al. 2009). Finally, a recently published meta-analysis of primary prevention ICD trials suggested that women may not benefit from implantation of the device (Ghanbari et al. 2009), adding further to the controversy of differential benefit in women versus men.

\section{The Study}

The investigators of the Ontario ICD Database recently published an analysis of sex differences among defibrillator patients (MacFadden et al. 2012). The analysis included patients who were referred for consideration of ICD implanta- 
tion in Ontario between February 2007 and July 2010. Data were prospectively collected at ICD implantation and follow-up centres by an electrophysiologist and trained research coordinators, who entered the information into a secure Web registry housed at the Institute for Clinical Evaluative Sciences (ICES).

This study examined the spectrum of patients beginning at the time of initial referral for consideration of a primary or secondary prevention ICD. The study was extended to the post-implantation period for early complications examined at 45 days and up to one year of follow-up. Complications were classified as either minor (not severe and did not require intervention to remedy) or major (severe and required intervention to remedy). The ICD outcomes, including the occurrence of appropriate ICD shocks (i.e., a potentially life-saving therapy delivered by the defibrillator) or appropriate antitachycardia therapy (i.e., a shock or pacing to treat ventricular tachycardia) were also examined, as were inappropriate shocks and death.

\section{Key Findings \\ ICD Implantation}

The study looked at 6,021 patients $(4,733$ men and 1,288 women) who were considered for an ICD by a cardiac electrophysiologist in Ontario. Of the 5,450 patients who were implanted with an ICD, 3,822 were implanted for primary and 1,628 for secondary prevention indications. Of those patients who were referred, the majority were accepted for an ICD: $89 \%$ of men and $87 \%$ of women for primary prevention indications, and $95 \%$ of men and $96 \%$ of women for secondary prevention indications. In an analysis where a relative risk (RR) of ICD implantation greater than one predicted increased probability of acceptance for the device in women, there was no evidence for differential utilization by sex. After adjustment for all potential clinical covariates, the RRs of ICD implantation were 0.99 (95\% confidence interval [CI] 0.96-1.02, $p=.61$ ) for primary prevention and 1.00 (95\% CI $0.98-1.03, p=.85$ ) for secondary prevention. Thus, there was no evidence for bias against women for ICD implantation after being referred to a cardiac electrophysiologist.

\section{Complications}

Of the study cohort, major early complications (within 45 days) occurred in 3.3\% of men and $5.4 \%$ of women $(p=.002)$, and the most common early complications were lead related - lead replacement in $1.9 \%$ of women and lead repositioning in $1.2 \%$ of men. Minor early complications occurred in $5.8 \%$ of women and $3.8 \%$ of men $(p=.006)$. The most commonly occurring early minor complication was lead related problems that did not require lead repositioning. After multivariable adjustment for patient clinical characteristics and co-morbidities, women were $78 \%$ more likely than men to experience a major early complication (95\% CI 1.24-2.58, $p=.002$ ). Women were also 55\% more likely to experience a minor early complication (95\% CI $1.09-2.20, p=.014)$.

When surveillance for complications was extended to one-year follow-up, the increased risk of complications in women was sustained. Examining 3,984 person-years of followup, the rates of major complications increased by $88 \%$ in women compared with men: 7.4 major complications per 100 personyears in men and 13.9 per 100 person-years in women (rate ratio $1.88,95 \%$ CI $1.50-2.36, p<.001)$. The most common causes of major complications in extended follow-up were lead repositioning, lead replacement, pocket infection and electrical storm. Minor complications in extended follow-up were $68 \%$ more likely to occur in women, with event rates of 6.2 events per 100 person-years in men and 10.4 per 100 person-years in women (rate ratio $1.68,95 \%$ CI $1.30-2.17, p<.001$ ). Frequent minor complications were lead dislodgements where leads were not repositioned, pocket hematomas and incisional infections.

\section{FIGURE 1.}

\section{Time to first appropriate and inappropriate shock, by sex}

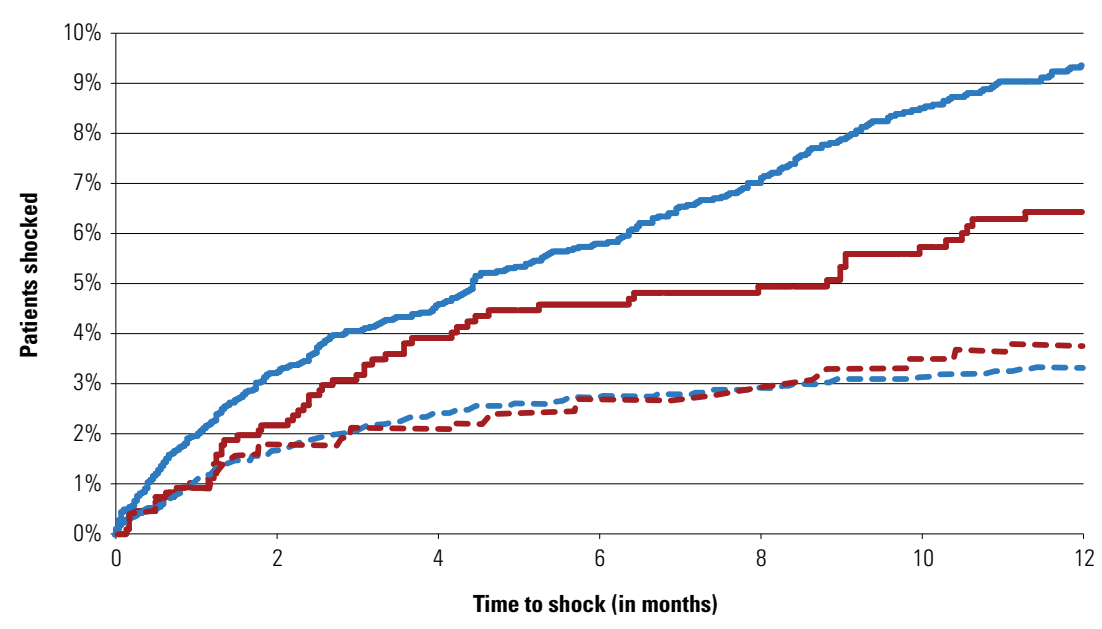

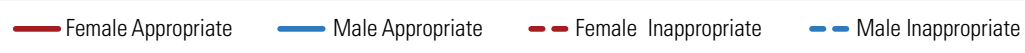




\section{Device Outcomes}

The cohort with device follow-up consisted of 5,213 ICD recipients, including 4,108 men and 1,105 women. The rate of appropriate shock per 100 person-years was increased in men at 10.5 compared with 7.2 in women $(\mathrm{P}=.009)$. Appropriate therapies were also increased in men compared with women: 21.3 versus 15.3 per 100 person-years $(P=.001)$. After multivariable adjustment for all clinical covariates, women were $31 \%$ less likely than men to experience an appropriate shock (hazard rate $[\mathrm{HR}] 0.69,95 \% \mathrm{CI} 0.51-0.93, \mathrm{P}=.015)$. Women were also $27 \%$ less likely to have any appropriate ICD therapy delivered (HR $0.73,95 \%$ CI $0.59-0.90, \mathrm{P}=.003$ ). The risk of inappropriate therapy was not significantly increased in women (HR $0.97,95 \%$ CI $0.64-1.47, \mathrm{P}=.89)$, and there was no significant difference in mortality (HR 1.00, 95\% CI 0.64-1.55, P = .99). Figure 1 shows the time to first appropriate and inappropriate shock for men and women.

\section{Bottom Line}

Women were less likely than men to experience an appropriate ICD shock or ICD-delivered therapy (i.e., a device shock or pacing therapy to treat rapid ventricular heart rhythms). In contrast, both major and minor complications from device implantation were higher in women. Finally, upon referral to a cardiac electrophysiologist, there were no differences between men and women in the rate of proceeding to ICD implantation after accounting for baseline differences in patient clinical characteristics.

\section{What Do These Findings Mean?}

The findings of this study indicate the following:

- Women are less likely than men to experience potentially lifesaving device therapy because electrical therapies delivered by the device occurred significantly less frequently in women. This study suggests that the current methods of selecting patients for an ICD may be better at identifying men who will benefit from the device than women. Improved methods are needed to identify women who are at risk for SCD so that women at lower risk are not needlessly implanted with an ICD.

- Women are more likely than men to experience both major and minor complications from ICD implantation, suggesting that greater care is needed peri-operatively. Improvements in ICD technology may provide incremental benefits to women, who are at higher risk of developing lead-related complications.

- Given the current patient selection protocols and device implantation methods, the net benefit of the ICD is reduced in women because of the lower rates of defibrillator therapies delivered and the increased risk of complications; this further underscores the importance of improved patient selection for this device.
- The higher numbers of ICDs implanted in men are not due to any appreciable differences in patient selection after referral to a cardiac electrophysiologist. The greater numbers of ICDs implanted in men may reflect biological differences in the risk of SCD or left ventricular function, or perhaps in upstream referral patterns.

\section{Future Research}

There is a need for more research into sex differences in the risk of ventricular arrhythmias. Additionally, future research should address whether antiarrhythmic technologies benefit women and men to the same degree. Randomized controlled trials

\section{Women were $\mathbf{3 1} \%$ less likely than men to experience an appropriate shock.}

of ICD therapies conducted in women only are also required to elucidate if there is a benefit of defibrillators in this group. However, prior to embarking on large randomized trials, there is an urgent need to identify risk stratification methods that incorporate patient sex as a key covariate in the analysis.

\section{Conclusion}

The current use of ICDs, applying similar inclusion criteria to both women and men, is associated with lower rates of defibrillator-delivered therapies in women. For women, lower rates of appropriate shock and appropriate therapy were observed, while they experienced higher complications during the first 45 days after implantation and up to one year of follow-up. Further research on the risk predictors of ventricular tachyarrhythmias and randomized trials of ICDs, including exclusively female cohorts, are needed. HQ

\section{Acknowledgements}

ICES is supported in part by a grant from the Ontario Ministry of Health and Long-Term Care (MOHLTC). The opinions, results and conclusions are those of the author, and no endorsement by ICES or MOHLTC is intended or should be inferred. This work was supported by an operating grant from the Canadian Institutes of Health Research (CIHR MOP 111150). Dr. Lee is a clinician-scientist of CIHR.

\section{References}

Albert, C.M., B.A. McGovern, J.B. Newell and J.N. Ruskin. 1996. "Sex Differences in Cardiac Arrest Survivors." Circulation 93(6): 1170-76.

Antiarrhythmics versus Implantable Defibrillators Investigators. 1997. "A Comparison of Antiarrhythmic-Drug Therapy with Implantable Defibrillators in Patients Resuscitated from Near-Fatal Ventricular Arrhythmias." New England Journal of Medicine 337(22): 1576-83.

Bardy, G.H., K.L. Lee, D.B. Mark, J.E. Poole, D.L. Packer, R. Boineau 
et al. 2005. "Amiodarone or an Implantable Cardioverter-Defibrillator for Congestive Heart Failure." New England Journal of Medicine 352(3): 225-37.

Chugh, S.S., A. Uy-Evanado, C. Teodorescu, K. Reinier, R. Mariani, K. Gunson et al. 2009. "Women Have a Lower Prevalence of Structural Heart Disease as a Precursor to Sudden Cardiac Arrest: The Ore-SUDS (Oregon Sudden Unexpected Death Study)." Journal of the American College of Cardiology 54(22): 2006-11.

Cupples, L.A., D.R. Gagnon and W.B. Kannel. 1992. "Long- and Short-Term Risk of Sudden Coronary Death." Circulation 85(1 Suppl.): I11-18.

Ghanbari, H., G. Dalloul, R. Hasan, M. Daccarett, S. Saba, S. David et al. 2009. "Effectiveness of Implantable Cardioverter-Defibrillators for the Primary Prevention of Sudden Cardiac Death in Women with Advanced Heart Failure: A Meta-Analysis of Randomized Controlled Trials." Archives of Internal Medicine 169(16): 1500-6.

Kannel, W.B., P.W. Wilson, R.B. D’Agostino and J. Cobb. 1998. "Sudden Coronary Death in Women." American Heart Journal 136(2): 205-12.

Lampert, R., C.A. McPherson, J.F. Clancy, T.L. Caulin-Glaser, L.E. Rosenfeld and W.P. Batsford. 2004. "Gender Differences in Ventricular Arrhythmia Recurrence in Patients with Coronary Artery Disease and Implantable Cardioverter-Defibrillators." Journal of the American College of Cardiology 43(12): 2293-99.

Lee, D.S., A.D. Krahn, J.S. Healey, D. Birnie, E. Crystal, P. Dorian et al. 2010. "Evaluation of Early Complications Related to De Novo
Cardioverter Defibrillator Implantation Insights from the Ontario ICD Database." Journal of the American College of Cardiology 55(8): 774-82.

MacFadden, D.R., E. Crystal, A.D. Krahn, I. Mangat, J.S. Healey, P. Dorian et al. 2012. "Sex Differences in Implantable CardioverterDefibrillator Outcomes: Findings from a Prospective Defibrillator Database." Annals of Internal Medicine 156(3): 195-203.

MacFadden, D.R., J.V. Tu, A. Chong, P.C. Austin and D.S. Lee. 2009. "Evaluating Sex Differences in Population-Based Utilization of Implantable Cardioverter-Defibrillators: Role of Cardiac Conditions and Noncardiac Comorbidities." Heart Rhythm 6(9): 1289-96.

Moss, A.J., W. Zareba, W.J. Hall, H. Klein, D.J. Wilber, D.S. Cannom et al. 2002. "Prophylactic Implantation of a Defibrillator in Patients with Myocardial Infarction and Reduced Ejection Fraction." New England Journal of Medicine 346(12): 877-83.

Peterson, P.N., S.L. Daugherty, Y. Wang, H.J. Vidaillet, P.A. Heidenreich, J.P. Curtis et al. 2009. "Gender Differences in ProcedureRelated Adverse Events in Patients Receiving Implantable CardioverterDefibrillator Therapy." Circulation 119(8): 1078-84.

\section{About the Author}

Douglas S. Lee, MD, PhD, is a scientist at the Institute for Clinical Evaluative Sciences in Toronto, Ontario. He may be contacted at dlee@ices.on.ca.

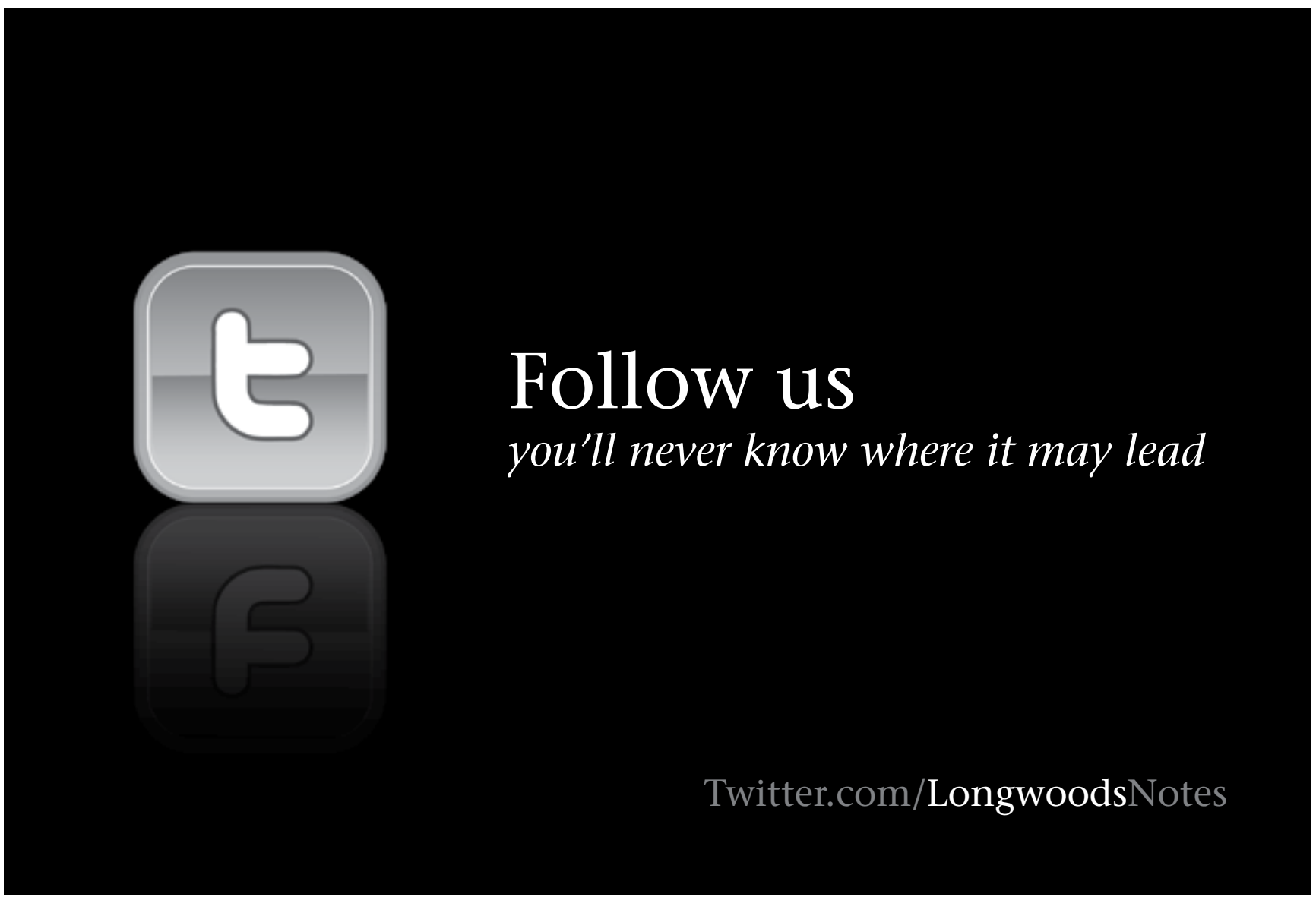

